\title{
Inverted internal limiting membrane flap technique versus complete internal limiting membrane peeling in large macular hole surgery: a comparative study
}

Prithvi Ramtohul ${ }^{1 *}\left(\mathbb{D}\right.$, Eric Parrat ${ }^{3}$, Danièle Denis ${ }^{1}$ and Umberto Lorenzi ${ }^{2}$

\begin{abstract}
Background: To compare the anatomical and functional outcomes of the inverted internal limiting membrane (ILM) flap technique and the complete ILM removal in the treatment of large stage 4 macular hole $(\mathrm{MH})>400 \mu \mathrm{m}$ and to evaluate reconstructive anatomical changes in foveal microstructure using spectral-domain optical coherence tomography.

Methods: This is a retrospective, consecutive, nonrandomized comparative study of patients affected by idiopathic, myopic or traumatic stage $4 \mathrm{MH}$ (minimum diameter $>400 \mu \mathrm{m}$ ) treated with 25-gauge pars-plana vitrectomy with either complete ILM peeling ( $n=23$, Group 1) or inverted ILM flap technique $(n=23$, Group 2), between August 2016 and August 2018. Main outcomes measured were the MH closure rate assessed by spectral-domain optical coherence tomography and the best-corrected visual acuity (BCVA) at six months. Foveal microstructure reconstructive changes were evaluated using SD-OCT to determine predictive factors of postoperative BCVA.

Results: Closure of MH was achieved in 16/23 cases of Group 1 (70\%) and in 22/23 cases of the Group 2 (96\%). Surgical failure was reported in 6 cases of Group 1 and 1 case of Group 2. The MH closure rate was significantly higher with the inverted ILM flap technique $(P$-value $=0.02)$. Average BCVA (LogMAR) changed from $1.04 \pm 0.32$ to $0.70 \pm 0.31$ in Group 1 and from $0.98 \pm 0.22$ to $0.45 \pm 0.25$ in Group 2 (P-value $=0.005)$ at 6 months. Improvement in BCVA (>0.3 LogMAR units) was statistically higher in the Group $2(P$-value $=0.03)$. Restoration of foveal microstructure was significantly higher in the Group 2 at 6 months (52\% vs 9\%, $P$-value $<0.01$ ). In Group 2, the integrity of the external limiting membrane at 3 months postoperatively was the only significant feature correlated with postoperative BCVA at 6 months ( $r=0.562$; $P$-Value $=0.01$, forward stepwise regression analysis).

Conclusion: Inverted ILM flap technique is more effective than the classic ILM peeling for the closure of large stage 4 $\mathrm{MHs}>400 \mu \mathrm{m}$, improving both anatomical and functional outcomes. Early recovery of the external limiting membrane at 3 months is a positive predictive value of postoperative BCVA 6 months after inverted ILM flap technique.
\end{abstract}

Keywords: Internal limiting membrane, Inverted ILM flap technique, ILM peeling, Macular hole, Vitrectomy

\footnotetext{
* Correspondence: pramtohul@free.fr

${ }^{1}$ Centre Hospitalier Universitaire de l'Hôpital Nord, chemin des Bourrely,

13015 Marseille, France

Full list of author information is available at the end of the article
}

(c) The Author(s). 2019 Open Access This article is distributed under the terms of the Creative Commons Attribution 4.0 International License (http://creativecommons.org/licenses/by/4.0/), which permits unrestricted use, distribution, and reproduction in any medium, provided you give appropriate credit to the original author(s) and the source, provide a link to the Creative Commons license, and indicate if changes were made. The Creative Commons Public Domain Dedication waiver (http://creativecommons.org/publicdomain/zero/1.0/) applies to the data made available in this article, unless otherwise stated. 


\section{Background}

A macular hole $(\mathrm{MH})$ is a retinal defect that arises at the level of the internal limiting membrane (ILM) and extends up to, but not including, the retinal pigment epithelium (RPE), in the foveolar region. Primary MH, resulting from vitreo-macular tractions, either tangential and anteroposterior, typically occurs in the elderly, with a prevalence ranging from $0,2 \%$ to $0,8 \%$ in the general population [1]. MH represents a vision-threatening event requiring a prompt treatment. MH surgery was first described by Kelly and Wendel in 1991 [2]. Ever since, progression of surgical techniques, instrumentations and diagnostic tools have enhanced the anatomical and functional outcomes of $\mathrm{MH}$ surgery.

Pars-plana vitrectomy (PPV) combined with ILM removal and gas tamponade is considered as the standard procedure in the treatment of $\mathrm{MH}$. Fundamental principles of the surgery efficacy are multifactorial and rest on the elimination of vitreo-macular tractional forces, the extensibility of the retina and the $\mathrm{MH}$ bridging by Müller cell gliosis activation [3].

Although the $\mathrm{MH}$ closure rate reaches approximately $90 \%$ with the standard procedure, some cases are more challenging and display a worsened prognosis [4]. In fact, large MHs' diameters $>400 \mu \mathrm{m}$, chronic MHs, and secondary $\mathrm{MHs}$, resulting from ocular trauma, severe myopia, retinal detachment or proliferative vitreoretinopathy, present with poor anatomical and functional outcomes [5]. In 2010, Michalewska et al. introduced the inverted ILM flap technique, improving both visual acuity and closure success of large idiopathic MHs and myopic MHs [6]. More recently, Rizzo et al. found out, in a large comparative study, a $95.6 \%$ and a $88.4 \%$ closure rate respectively in idiopathic $\mathrm{MHs}>400 \mu \mathrm{m}$ and myopic MHs with the inverted ILM flap procedure [7].

Several studies reported spectral-domain optical coherence tomography (SD-OCT) structural changes in the photoreceptor layers of eyes with surgically closed $\mathrm{MH}$, including disruption of the ellipsoid zone (EZ) and external limiting membrane (ELM). However, only a few studies evaluated the reconstructive change in foveal anatomy in MHs surgically closed with the inverted ILM flap technique, suggesting that the restoration of the ELM is a critical structural feature significantly correlated with improved postoperative BCVA [8].

The purpose of this study was to compare the anatomical and functional outcomes of the inverted ILM flap technique and the complete ILM removal in the treatment of large stage $4 \mathrm{MHs}$ (diameter $>400 \mu \mathrm{m}$ ) and to evaluate reconstructive anatomical changes in foveal microstructure using SD-OCT.

\section{Methods}

This is a retrospective study based on the review of medical records of forty-six eyes of 46 consecutive patients affected by large stage $4 \mathrm{MH}$, who underwent a surgical treatment at Clinique Les Eaux Claires and University Medical Center of Pointe-à-Pitre in Guadeloupe between August 2016 and August 2018. The study complied with the Declaration of Helsinski. Ethical committee approval was not required due to the retrospective nature of this study. Oral informed consent for participation was obtained from all patients.

The inclusion criteria were as follows: minimum $\mathrm{MH}>$ $400 \mu \mathrm{m}$, age $>18$ and at least 6 months of follow-up. Exclusion criteria were the presence of a chorioretinal atrophy involving the fovea (diagnosed on SD-OCT as a [1] region of hypertransmission of at least $250 \mu \mathrm{m}$ in diameter, [2] a zone of attenuation or disruption of the RPE of at least $250 \mu \mathrm{m}$ in diameter, [3] evidence of overlying photoreceptor degeneration, and [4] absence of scrolled RPE or other signs of an RPE tear), previous retinal surgery, presence of choroidal neovascularization, diabetic retinopathy or other ocular conditions that could influence the BCVA, except lens opacity.

At baseline and at every follow-up visit (1 month, 3 months and 6 months after surgery), all patients underwent a complete ophthalmologic examination, including measurement of the BCVA, slit-lamp examination and biomicroscopy of the posterior segment using a slitlamp with a non-contact 90D lens. BCVA measured with a Snellen chart was converted to logarithm of minimum angle of resolution (LogMAR) for statistical analysis. $\mathrm{MH}$ diameter and outer retinal layers were evaluated at the same time by SD-OCT (Heidelberg Engineering $\mathrm{GmbH}$, Heidelberg, Germany). Tracking system was used for the follow-up, allowing accurate and repeatable measurements.

Microstructural imaging analysis of the fovea was performed using SD-OCT. Restoration of the photoreceptor layer was evaluated as recovery of the continuous backreflection lines corresponding to the EZ and the ELM. We defined the continuous restoration of the EZ and the ELM as EZ (+) and ELM (+) respectively. The postoperative photoreceptor recovery, corresponding to the EZ and the ELM lines, was evaluated at every follow-up visit (1 month, 3 months, 6 months). Two masked investigators (R.R. and T.A.) evaluated the SD-OCT images. In case of disagreement, a third investigator (M.S.) was referred for final decision.

PPV with conventional ILM peeling combined with C2F6 gas (perfluoroethane) tamponade was conducted in 23 eyes (Group 1: ILM peeling group) between August 2016 and August 2017, while PPV with inverted ILM flap technique and C2F6 gas tamponade was performed in 23 eyes (Group 2: ILM flap group) between August 2017 and August 2018. Cataract surgery was simultaneously performed in 13 eyes ( 7 eyes in the Group 1 and 6 eyes in the Group 2). 
All patients had been operated by one of two experienced vitreoretinal surgeons (U.L. and E.P.).

\section{Surgical techniques Complete ILM removal}

A complete pars-plana vitrectomy was performed using the 25-gauge Stellaris PC System (Bausch and Lomb, Rochester, NY, USA), sutureless, and the Ocular Landers Wide Field Vitrectomy Lens (Ocular Instruments, Bellevue, Washington, USA). After this, the ILM was stained with ILM Blue injection (DORC, Aqumen Biopharmaceutical KK, Fukuoka, Japan) above the macula, followed by lavage. The ILM was peeled off centripetally, approximately 2 disk diameters around the $\mathrm{MH}$. At the end of the surgery, the eye was filled with perfluoroethane (C2F6, 16\%). The patients were instructed to maintain a face-down position for 5 days.

\section{ILM flap technique}

It was performed according to the original report of Michalewska et al [6]. During ILM peeling around the $\mathrm{MH}$, the ILM flap was not entirely removed from the retina but a remnant was left attached to the edges of the MH. The ILM flap was approximately 1 or 1.5 diameter of the $\mathrm{MH}$. It was lifted, inverted and pushed into the $\mathrm{MH}$ gently with forceps (25-gauge Eckardt Endgripping Forceps, Dutch Ophtalmic, USA) to fill the entire $\mathrm{MH}$. Fluid-air exchange was performed using a low intraocular pressure and passive aspiration to avoid turbulence that could cause a flap displacement. At the end of the surgery, the eye was filled with perfluoroethane (C2F6, 16\%). The patients were instructed to maintain a face-down position for 5 days.

\section{Statistical analysis}

Statistical analysis was performed using JMP statistical analysis software, version 5.01 J (SAS Institute, Cary, $\mathrm{NC)}$ to analyse the differences in baseline characteristics, anatomical outcomes including macular hole closure and functional outcomes of BCVA between ILM peeling group (Group 1) and ILM flap group (Group 2). The BCVA was recorded as decimal value and converted to LogMAR for statistical analysis.

The Wilcoxon rank test was used to compare pre- and postoperative BCVA. The Chi2-test was used to compare $\mathrm{MH}$ closure rates after surgery and improvement in BCVA (>0,3 LogMAR units) between 2 groups. The comparison of BCVA in the two groups was analysed in the Mann-Whitney U-test. Kruskal-Wallis 1-way analysis of variance (ANOVA) was performed followed by pairwise multiple comparison using Dunn's method to assess the association between visual outcomes and the degree of recovery of the photoreceptor layer. The sample size was determined assuming that the inverted ILM flap technique was more effective for achieving large $\mathrm{MH}$ closure than classic ILM peeling (95\% vs $85 \%$ ) based on literature data. A sample size of 60 eyes was required to power the study comparison to $80 \%$ (with a $95 \%$ confidence interval). A $P$-value $<0.05$ was considered to be statistically significant.

\section{Results}

Twenty-three eyes of 23 consecutive patients operated with ILM removal (Group 1) and twenty-three eyes of 23 consecutive patients treated with inverted ILM flap technique (Group 2) were included in this study. Patients' characteristics are shown in Table 1.

The comparison of preoperative data between the two groups are shown in Table 2 . There were no statistically significant differences in age ranges $(P$-value $=0.14)$, sex $(P$-value $=0.37)$, duration of symptoms $(P$-value $=0.54)$, type of $\mathrm{MH}$ (idiopathic: $P$-value $=0.75 /$ myopic: $P$-value $=$ 0.44 /traumatic: $P$-value $=0.64$ ) or preoperative BCVA $(P$-value $=0.48)$. The mean minimal $\mathrm{MH}$ diameter was higher in the inverted ILM flap group, but the difference was not statistically significant (Peeling group: $574.09 \pm$ $164.68 \mu \mathrm{m}$; ILM flap group: $657.33 \pm 172.36 \mu \mathrm{m}, \quad P$ value $=0.09$ ).

Surgery was considered successful when a complete closure of the $\mathrm{MH}$ was achieved (defined as the absence of a neurosensory defect over the retina). Flat-open and elevated-open $\mathrm{MH}$ were considered surgical failures. At six months of follow-up, SD-OCT showed MH closure in 16 cases in Group 1 (70\%) and 21 cases in Group 2 (91\%) after one single surgery. No cases of flat-open or elevated-open $\mathrm{MH}$ were found at 6 months.

Seven surgical failures occurred in the Group 1. The mean diameter of the $\mathrm{MH}$ was $643.83 \pm 157.37 \mu \mathrm{m}$. An additional surgery was performed for three patients but the $\mathrm{MH}$ remained open at 6 months. In Group 2, there were 2 surgical failures (Mean diameter $=573 \mu \mathrm{m} \pm$ $2 \mu \mathrm{m})$. One of these patients was reoperated and presented a $\mathrm{MH}$ closure after a 6-month follow-up.

Phacoemulsification was performed in combination with PPV in 7 cases (30\%) from the Group 1 and 6 cases (26\%) from the Group 2. Therefore, each patient included was pseudophakic at the end of the follow-up.

Postoperative data are shown in Table 3. No statistically significant differences were found in the postoperative BCVA at 1 and 3 months ( $P$-value $>0.05$ respectively). Mean BCVA at 6 months was $0.70 \pm 0.31$ LogMAR in the ILM peeling group and $0.45 \pm 0.25$ LogMAR in the ILM flap group $(P$-value $<0.01)$. The difference between preoperative and postoperative BCVA was statically significant within the Group 1 and the Group 2 $(P$-value $<0.001)$. The improvement in BCVA $(>0.3$ LogMAR units) at 6 months was significantly higher in the ILM flap group $(P$-value $=0.03)$. 
Table 1 Patient characteristics. Group 1: ILM Removal Group and Group 2: Inverted ILM flap Group

\begin{tabular}{|c|c|c|c|c|c|c|c|}
\hline Patient no. & Age range & Eye & Preoperative lens status & MH diameter $(\mu \mathrm{m})$ & Operative procedure & Tamponade & Preoperative BCVA (decimal) \\
\hline \multicolumn{8}{|l|}{ Group 1} \\
\hline 1 & $70-74$ & R & $\mathrm{IOL}$ & 598 & PPV, PL & C2F6 & 0.2 \\
\hline 2 & $75-79$ & $\mathrm{R}$ & $\mathrm{IOL}$ & 652 & PPV, PL & $\mathrm{C} 2 \mathrm{~F} 6$ & 0.01 \\
\hline 3 & $75-79$ & L & $\mathrm{IOL}$ & 479 & PPV, PL & $\mathrm{C} 2 \mathrm{~F} 6$ & 0.2 \\
\hline 4 & $60-64$ & R & Phake & 753 & PPV, PL, PEA, IOL & $\mathrm{C} 2 \mathrm{~F} 6$ & 0.1 \\
\hline 5 & $75-79$ & $\mathrm{R}$ & $\mathrm{IOL}$ & 451 & PPV, PL & C2F6 & 0.1 \\
\hline 6 & $65-69$ & L & $\mathrm{IOL}$ & 505 & PPV, PL & $\mathrm{C} 2 \mathrm{~F} 6$ & 0.2 \\
\hline 7 & $55-59$ & L & $\mathrm{IOL}$ & 697 & PPV, PL & C2F6 & 0.05 \\
\hline 8 & $60-64$ & $\mathrm{R}$ & $\mathrm{IOL}$ & 500 & PPV, PL & $\mathrm{C} 2 \mathrm{~F} 6$ & 0.2 \\
\hline 9 & $65-69$ & L & Phake & 1028 & PPV, PL, PEA, IOL & $\mathrm{C} 2 \mathrm{~F} 6$ & 0.05 \\
\hline 10 & $70-74$ & R & Phake & 479 & PPV, PL, PEA, IOL & $\mathrm{C} 2 \mathrm{~F} 6$ & 0.15 \\
\hline 11 & $65-69$ & L & Phake & 401 & PPV, PL, PEA, IOL & $\mathrm{C} 2 \mathrm{~F} 6$ & 0.2 \\
\hline 12 & $70-74$ & R & $\mathrm{IOL}$ & 603 & PPV, PL & $\mathrm{C} 2 \mathrm{~F} 6$ & 0.1 \\
\hline 13 & $60-64$ & R & $\mathrm{IOL}$ & 401 & PPV, PL & $\mathrm{C} 2 \mathrm{~F} 6$ & 0.05 \\
\hline 14 & $70-74$ & L & $\mathrm{IOL}$ & 654 & PPV, PL & $\mathrm{C} 2 \mathrm{~F} 6$ & 0.2 \\
\hline 15 & $75-79$ & L & $\mathrm{IOL}$ & 400 & $P P V, P L$ & $\mathrm{C} 2 \mathrm{~F} 6$ & 0.1 \\
\hline 16 & $50-54$ & R & Phake & 879 & PPV, PL, PEA, IOL & $\mathrm{C} 2 \mathrm{~F} 6$ & 0.05 \\
\hline 17 & $60-64$ & $\mathrm{R}$ & $\mathrm{IOL}$ & 695 & PPV, PL & $\mathrm{C} 2 \mathrm{~F} 6$ & 0.05 \\
\hline 18 & $65-69$ & $\mathrm{R}$ & $\mathrm{IOL}$ & 450 & PPV, PL & $\mathrm{C} 2 \mathrm{~F} 6$ & 0.05 \\
\hline 19 & $60-64$ & L & $\mathrm{IOL}$ & 508 & PPV, PL & $\mathrm{C} 2 \mathrm{~F} 6$ & 0.1 \\
\hline 20 & $70-74$ & R & $\mathrm{IOL}$ & 400 & PPV, PL & $\mathrm{C} 2 \mathrm{~F} 6$ & 0.15 \\
\hline 21 & $65-69$ & R & Phake & 400 & PPV, PL, PEA, IOL & $\mathrm{C} 2 \mathrm{~F} 6$ & 0.1 \\
\hline 22 & $65-69$ & R & $\mathrm{IOL}$ & 650 & PPV, PL & $\mathrm{C} 2 \mathrm{~F} 6$ & 0.05 \\
\hline 23 & $60-64$ & R & Phake & 621 & PPV, PL, PEA, IOL & C2F6 & 0.1 \\
\hline \multicolumn{8}{|l|}{ Group 2} \\
\hline 1 & $65-69$ & L & $\mathrm{IOL}$ & 426 & PPV, ILM & $\mathrm{C} 2 \mathrm{~F} 6$ & 0.12 \\
\hline 2 & $70-74$ & L & $\mathrm{IOL}$ & 432 & PPV, ILM & C2F6 & 0.3 \\
\hline 3 & $65-69$ & $R$ & Phake & 502 & PPV, ILM, PEA, IOL & $\mathrm{C} 2 \mathrm{~F} 6$ & 0.16 \\
\hline 4 & $65-69$ & $\mathrm{R}$ & $\mathrm{IOL}$ & 571 & PPV, ILM & $\mathrm{C} 2 \mathrm{~F} 6$ & 0.1 \\
\hline 5 & $65-69$ & R & $\mathrm{IOL}$ & 575 & PPV, ILM & $\mathrm{C} 2 \mathrm{~F} 6$ & 0.08 \\
\hline 6 & $70-74$ & L & $\mathrm{IOL}$ & 573 & PPV, ILM & $\mathrm{C} 2 \mathrm{~F} 6$ & 0.1 \\
\hline 7 & $75-79$ & $\mathrm{R}$ & Phake & 593 & PPV, ILM, PEA, IOL & C2F6 & 0.16 \\
\hline 8 & $65-69$ & L & $\mathrm{IOL}$ & 640 & PPV, ILM & C2F6 & 0.04 \\
\hline 9 & $70-74$ & R & $\mathrm{IOL}$ & 642 & PPV, ILM & $\mathrm{C} 2 \mathrm{~F} 6$ & 0.16 \\
\hline 10 & $60-64$ & R & $\mathrm{IOL}$ & 694 & PPV, ILM & C2F6 & 0.08 \\
\hline 11 & $65-69$ & $L$ & $\mathrm{IOL}$ & 699 & PPV, ILM & $\mathrm{C} 2 \mathrm{~F} 6$ & 0.08 \\
\hline 12 & $60-64$ & L & Phake & 691 & PPV, ILM, PEA, IOL & $\mathrm{C} 2 \mathrm{~F} 6$ & 0.04 \\
\hline 13 & $65-69$ & R & Phake & 739 & PPV, ILM, PEA, IOL & $\mathrm{C} 2 \mathrm{~F} 6$ & 0.08 \\
\hline 14 & $45-49$ & L & $\mathrm{IOL}$ & 746 & PPV, ILM & $\mathrm{C} 2 \mathrm{~F} 6$ & 0.16 \\
\hline 15 & $75-79$ & L & Phake & 798 & PPV, ILM, PEA, IOL & $\mathrm{C} 2 \mathrm{~F} 6$ & 0.1 \\
\hline 16 & $60-64$ & $\mathrm{R}$ & $\mathrm{IOL}$ & 792 & PPV, ILM & $\mathrm{C} 2 \mathrm{~F} 6$ & 0.16 \\
\hline 17 & $75-79$ & L & $\mathrm{IOL}$ & 833 & PPV, ILM & $\mathrm{C} 2 \mathrm{~F} 6$ & 0.16 \\
\hline 18 & $70-74$ & R & $\mathrm{IOL}$ & 891 & PPV, ILM & $\mathrm{C} 2 \mathrm{~F} 6$ & 0.1 \\
\hline 19 & $40-44$ & L & $\mathrm{IOL}$ & 1159 & PPV, ILM & $\mathrm{C} 2 \mathrm{~F} 6$ & 0.1 \\
\hline
\end{tabular}


Table 1 Patient characteristics. Group 1: ILM Removal Group and Group 2: Inverted ILM flap Group (Continued)

\begin{tabular}{clllllll}
\hline Patient no. & Age range & Eye & Preoperative lens status & MH diameter $(\mu \mathrm{m})$ & Operative procedure & Tamponade & Preoperative BCVA (decimal) \\
\hline 20 & $55-59$ & $\mathrm{R}$ & Phake & 674 & PPV, ILM, PEA, IOL & C2F6 & 0.05 \\
21 & $75-79$ & $\mathrm{R}$ & IOL & 721 & PPV, ILM & C2F6 & 0.1 \\
22 & $55-59$ & $\mathrm{R}$ & IOL & 438 & PPV, ILM & C2F6 & 0.25 \\
23 & $65-69$ & R & IOL & 524 & PPV, ILM & C2F6 & 0.1 \\
\hline
\end{tabular}

Abbreviations: BCVA best-corrected visual acuity, C2F8 hexafluoroethane, ILM Inverted ILM flap, IOL intraocular lens, LogMAR logarithm of minimum angle of resolution, $M H$ macular hole, $P E A$ phacoemulsification, $P P V$ pars plana vitrectomy

The primary $\mathrm{MH}$ closure rate was $70 \%(16 / 23)$ in Group 1 and 91\% $(21 / 23)$ in Group 2 ( $P$-value $=0.67)$. The final $\mathrm{MH}$ closure rates at 6 months remained unchanged at $70 \%(16 / 23)$ in Group 1 . However, it reached $96 \%(22 / 23)$ in Group 2 and this difference was statistically significant $(\mathrm{P}$-value $=0.02)$.

Postoperative recovery of the photoreceptor layers is shown in Table 4. SD-OCT examination in the Group 1 revealed ELM (+) in $0(0 \%), 2(9 \%), 4(17 \%)$ patients at 1,3 and 6 months respectively, and EZ (+) in $0(0 \%), 1$ (4\%), 2 (9\%) patients at 1,3 and 6 months respectively. In Group 2, the ELM (+) was seen in 2 (9\%), 12 (52\%), $15(65 \%)$ patients at 1,3 and 6 months respectively and the EZ (+) was found in 1 (4\%), 5 (21\%), 12 (52\%) patients at 1,3 and 6 months respectively. Complete EZ restoration was not observed without complete restoration of the ELM. In both groups, restoration of the ELM preceded restoration of the EZ. Restoration of both ELM and EZ was significantly higher in the Group 2 (52\% vs $9 \%, P$-value $<0.01)$. Figure 1 highlights a case of complete ELM and EZ recovery following inverted ILM flap technique.

In Group 2, the integrity of the ELM at 3 months postoperatively was the only significant feature correlated with postoperative BCVA at 6 months $(r=0.562 ; P$ Value $=0.01$, forward stepwise regression analysis).

\section{Discussion}

Vitrectomy for $\mathrm{MH}$, first described by Kelly and Wendel in 1991, has been the golden standard [2]. However, its effectiveness may be impaired in case of large, chronic or myopic MHs. Michalewska et al. proposed a novel surgical management, called inverted ILM flap technique, and demonstrated its efficacy in large idiopathic MHs (diameter $>400 \mu \mathrm{m}$ ) and in myopic MHs, improving both anatomical and functional outcomes [6].

In the current study, we included patients affected by idiopathic, myopic or traumatic stage $4 \mathrm{MHs}$. Our results showed that PPV associated with inverted ILM flap technique and gas tamponade displays a statistically higher closure rate $(96 \%$ in the inverted ILM Group versus $70 \%$ in the ILM peeling Group, $P$-value $<0.05)$. Visual outcomes were also relevant in our study, as final BCVA was significantly higher with the inverted ILM flap technique $(P$-value $<0.01)$.

The exact mechanism of improved surgical results using the inverted ILM flap technique is not precisely understood. One theory is that the ILM flap may act as a scaffold for glial cells proliferation, resulting in $\mathrm{MH}$

Table 2 Comparison of preoperative study participant data

\begin{tabular}{llll}
\hline Characteristics & Group 1 (ILM Removal) & Group 2 (Inverted ILM Flap) & $P$-value \\
\hline No. of eyes & 23 & 23 & $68.03 \pm 9.50$ \\
Age (years; mean \pm SD) & $65.69 \pm 10.25$ & $13(56 \%) / 10(44 \%)$ & 0.14 \\
Gender (male/female) & $16(69 \%) / 7(31 \%)$ & $12.5 \pm 4.1$ & \\
Duration of symptoms (weeks; mean \pm SD) & $13.2 \pm 3.6$ & $6(26 \%)$ \\
Preoperative lens status, $n$ (\%) & & $17(74 \%)$ & 0.37 \\
Phakic & $7(30 \%)$ & $0.98 \pm 0.22$ & $657.33 \pm 172.36$ \\
Pseudophakic & $16(70 \%)$ & & 0.75 \\
Preoperative BCVA (LogMAR; mean \pm SD) & $1.04 \pm 0.32$ & 16 & 0.75 \\
MH diameter ( $\mu$ m; mean \pm SD) & $574.09 \pm 164.68$ & 5
\end{tabular}

Abbreviations: BCVA best-corrected visual acuity, LogMAR logarithm of minimum angle of resolution, $M H$ macular hole 
Table 3 Comparison of postoperative study participant data

\begin{tabular}{llll}
\hline Characteristics & Group 1 (ILM Removal) & Group 2 (Inverted ILM Flap) & $P$-value \\
\hline Postoperative BCVA (LogMAR; mean \pm SD) & & & $0.74 \pm 0.25$ \\
1 month & $0.77 \pm 0.31$ & $0.67 \pm 0.26$ & 0.73 \\
3 months & $0.67 \pm 0.33$ & $0.45 \pm 0.25$ & 0.95 \\
6 months & $0.70 \pm 0.31$ & $-0.54 \pm 0.25$ & 0.005 \\
Improvement in BCVA & $-0.35 \pm 0.33$ & $91 \%(21 / 23)$ & 0.03 \\
Primary MH closure rate & $70 \%(16 / 23)$ & 1 (One surgical success) & 0.67 \\
'Additional surgery & 3 (No surgical success) & $96 \%(22 / 23)$ & 0.30 \\
Final MH closure rate & $70 \%(16 / 23)$ & 0.02 \\
\hline
\end{tabular}

Abbreviations $B C V A$ best-corrected visual acuity, LogMAR logarithm of minimum angle of resolution, $M H$ macular hole

filling with proliferating cells, which enhances $\mathrm{MH}$ closure. Another explanation might be that the ILM serves as a barrier, disabling the entrance of fluid from the vitreous cavity to the MH [9]. Moreover, the ILM flap is a source of Müller cells, which located at the surface of the ILM flap. Müller cells are important for assuming the role of optical fibers to transfer light from retinal surface to the photoreceptor cell layer. These findings explain why inducing Müller cells proliferation improves not only the $\mathrm{MH}$ closure rate but also the postoperative visual acuity [10].

Our findings are consistent with recent literature: Michalewska et al. [6], Kuriyama et al. [11], Mete et al. [12], and Ota et al. [13], found similar anatomical and functional outcomes for myopic MHs. In a recent comparative study conducted in a large series of patients, Rizzo et al. demonstrated the effectiveness of the inverted ILM flap technique compared with the ILM peeling for idiopathic and myopic MHs, improving both anatomical and visual outcomes. Among the patients affected by full-thickness MHs (diameter $>400 \mu \mathrm{m}$ ), the closure rate was $95.6 \%$ in the inverted ILM flap group and the mean postoperative BCVA was 0.43 LogMAR $(P$-value $<0.01)[7]$. Our results are consistent with this study.

In our study, reconstruction of the foveal ELM 3 months postoperatively helps predict subsequent restoration of the ellipsoid zone and the potential for better visual outcomes. Restoration of both ELM and EZ was significantly higher with the inverted ILM flap technique. Integrity of the ELM at 3 months postoperatively was correlated with postoperative BCVA at 6 months $(r=0.562 ; P$-Value $=0.01$, forward stepwise regression analysis). Our results are consistent with Wakabayashi et al. suggesting that the ELM is a critical structural feature significantly correlated with postoperative BCVA [14].

Alternative surgical techniques have been proposed, trying to improve the original procedure described by Michalewska et al. Casini et al. compared the classic ILM flap technique with a modified and shorter ILM flap technique: the ILM flap remains attached to the retina only to the edges of the $\mathrm{MH}$, looking like a funnel with the narrow opening attached to the retina, and no extra manipulations of the flap were performed. Their results showed no significantly difference in anatomical and functional outcomes between these two techniques [15]. Rossi et al. reported two surgical variations of the original ILM flap technique. In the "Cover technique", the ILM was peeled centripetally and placed onto the $\mathrm{MH}$ in order to bridge the entire retinal defect with a single layer. In the "Fill technique", the ILM was folded in multiple layers and packed within the $\mathrm{MH}$ using forceps. These two techniques showed similar closure rates and postoperative vision at 3 months. According to the authors, the Fill technique could be more efficient in closing larger $\mathrm{MH}$ [16]. Michalewska et al. reported a comparative study to determine if a temporal inverted ILM flap is as effective as the classic ILM flap technique for the repair of large stage IV idiopathic MH. They described a modified form where ILM peeling is restricted to the temporal side of the fovea only; the $\mathrm{MH}$ is then covered with the temporal ILM flap. Their results showed no significant differences in initial and final

Table 4 Photoreceptor restoration during the follow-up period

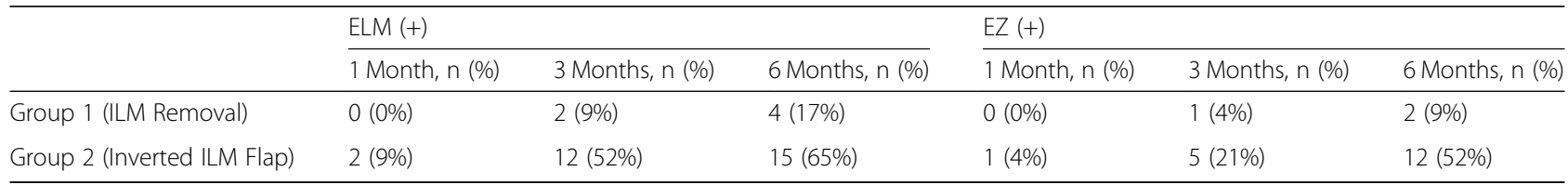

Abbreviations: $E L M(+)$ Restoration of the external limiting membrane, $E Z$ (+) Restoration of the ellipsoid zone, ILM Internal limiting membrane 


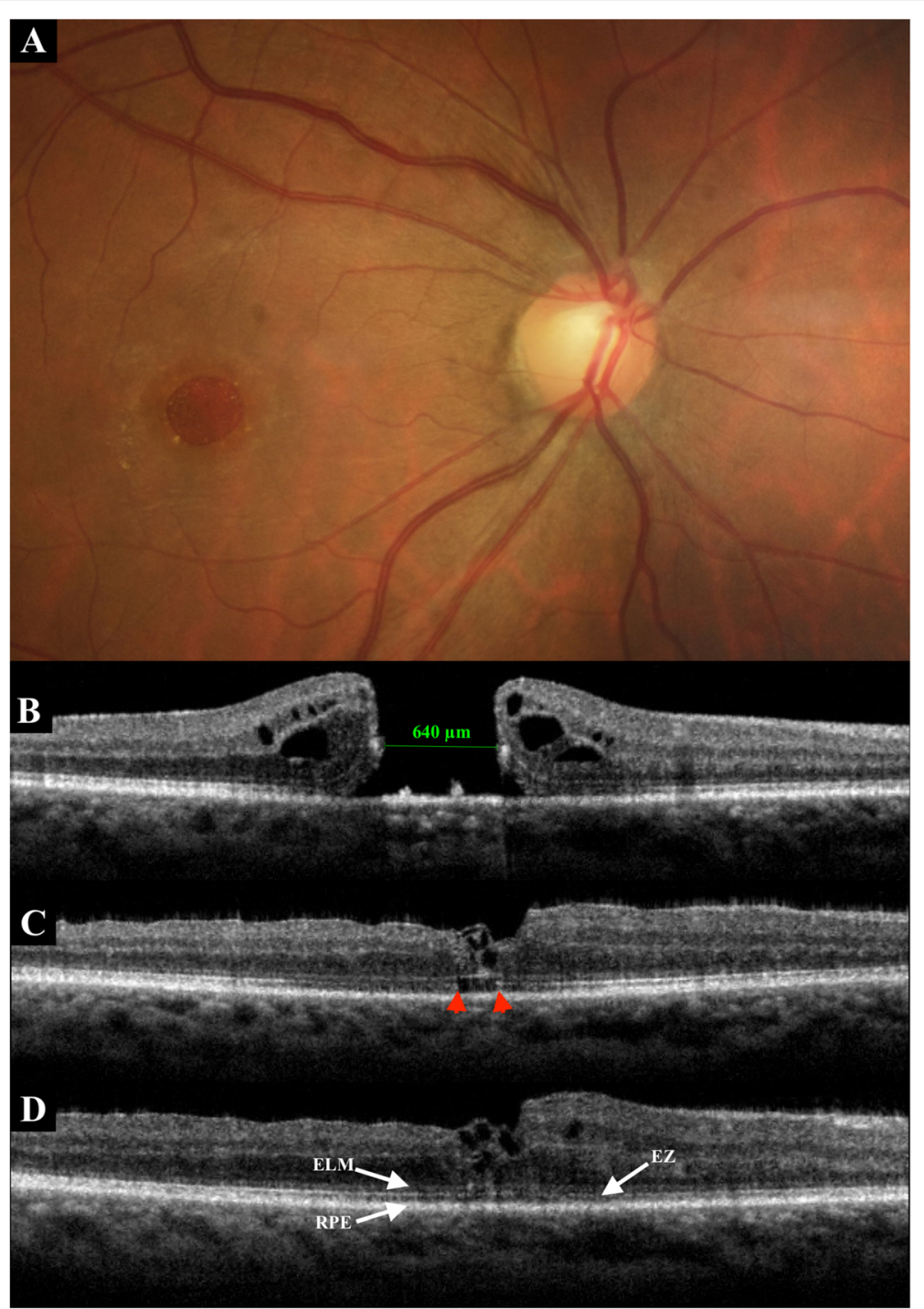

Fig. 1 A 61-year-old man presented with idiopathic large MH. a. Fundus photography before surgery. b. Baseline SD-OCT. The minimum diameter was $640 \mu \mathrm{m}$. Preoperative BCVA was 0.04 . c. SD-OCT at 1 month postoperatively. Inverted ILM flap technique was performed, with a W-shape closure (irregular closure) of the MH. SD-OCT showed the foveal hyperreflective tissue corresponding to a coiling of the flap. Disruption of the EZ (between red arrowheads) remained. The ELM line was almost complete. BCVA was 0.16. d. Six months after surgery, SD-OCT showed a persistent W-shape closure of the MH with a wrapped flap. There was a complete restoration of the ELM and EZ lines. The BCVA increased to 0.5

visual acuities between both techniques. The MH closure rate was $100 \%$ in both groups. They also noticed that the temporal inverted flap technique is associated with a lower incidence of dissociated optic nerve fiber layer (DONFL) by minimizing unnecessary surgical trauma to the nerve fiber layer [17]. Although DONFL has not been definitely associated with a decrease of visual acuity or microperimetry changes [18], there may be unknown effects on function that are not yet known. Temporal inverted ILM flap technique achieves satisfactory anatomical and functional results, as well as reduction of
DONFL appearance, especially in the area of papillomacular bundle.

Our study has several limitations, including its retrospective nature, the absence of randomization for the type of treatment chosen, the relatively small number of patients and the follow-up period of 6 months, which was relatively short to evaluate the final BCVA. The power of our study could have been insufficient, considering the number of included patients, however, it was enough to draw a definitive conclusion with statistically significant results. This might suggest the clear 
superiority of the inverted ILM flap technique in the treatment of large MHs.

\section{Conclusions}

In summary, inverted ILM flap technique is more effective than the classic ILM peeling for the closure of large stage $4 \mathrm{MHs}>400 \mu \mathrm{m}$, improving both anatomical and functional outcomes. We assessed foveal microstructural reconstructive changes following macular hole surgery. Restoration of both ELM and EZ was significantly higher with the inverted ILM flap technique. Early postoperative recovery of the ELM acts as a positive predictive value of final postoperative BCVA after inverted ILM flap technique.

\section{Acknowledgements}

None.

\section{Authors' contribution}

PR wrote the article. EP, DD and UL performed surgeries and follow-up All authors read and approved the final manuscript.

\section{Funding}

None.

\section{Availability of data and materials}

applicable. All data generated or analysed during the current study are included in this published article.

\section{Ethics approval and consent to participate}

applicable. It was performed in accordance with the tenets of the Declaration of Helsinki. No administrative permissions were needed to access the raw data. The Ethic committee of the faculty Timone (University of Medicine Aix-Marseille), Marseille, France, approved the study.

\section{Consent for publication}

applicable. The patients gave oral permission because written consent was not required by the institutional ethic committee. This report does not contain any personal information that could lead to the identification of the patients.

\section{Competing interests}

The authors declare that they have no competing interests.

\section{Author details}

${ }^{1}$ Centre Hospitalier Universitaire de l'Hôpital Nord, chemin des Bourrely, 13015 Marseille, France. ${ }^{2}$ Centre Hospitalier Universitaire de Rouen, 37 Boulevard Gambetta, 76000 Rouen, France. ${ }^{3}$ Department of Ophthalmology, University Medical Center of Pointe-à-Pitre, Les Abyme, Guadeloupe.

Received: 24 March 2019 Accepted: 27 December 2019

Published online: 06 January 2020

\section{References}

1. Boninska K, Nawrocki J, Michalewska Z. Mechanism of "flap closure" after the inverted internal limiting membrane flap technique. Retina. 2018;38(11): 2184-2189.

2. Kelly NE, Wendel RT. Vitreous surgery for idiopathic macular holes. Results of a pilot study. Arch Ophthalmol. 1991;109(5):654-9.

3. Yuan J, Zhang L-L, Lu Y-J, Han M-Y, Yu A-H, Cai X-J. Vitrectomy with internal limiting membrane peeling versus inverted internal limiting membrane flap technique for macular hole-induced retinal detachment: a systematic review of literature and meta-analysis. BMC Ophthalmoly. 2017;17(1):219. https://doi.org/10.1186/s12886-017-0619-8.

4. Okuda T, Higashide T, Kobayashi K, Ikuno Y, Sugiyama K. MACULAR HOLE CLOSURE OVER RESIDUAL SUBRETINAL FLUID BY AN INVERTED INTERNAL LIMITING MEMBRANE FLAP TECHNIQUE IN PATIENTS WITH MACULAR HOLE
RETINAL DETACHMENT IN HIGH MYOPIA. Retin Cases Brief Rep. 2016;10(2): 140-4. https://doi.org/10.1097//CB.0000000000000205.

5. Medina CA, Ortiz AG, Relhan N, Smiddy WE, Townsend JH, Flynn HW. Macular hole after pars Plana Vitrectomy for Rhegmatogenous retinal detachment. Retina. 2017;37(6):1065-72. https://doi.org/10.1097/IAE. 0000000000001351

6. Michalewska Z, Michalewski J, Adelman RA, Nawrocki J. Inverted internal limiting membrane flap technique for large macular holes. Ophthalmol. 2010;117(10):2018-25. https://doi.org/10.1016/j.ophtha.2010.02.011.

7. Rizzo S, Tartaro R, Barca F, Caporossi T, Bacherini D, Giansanti F. INTERNAL LIMITING MEMBRANE PEELING VERSUS INVERTED FLAP TECHNIQUE FOR TREATMENT OF FULL-THICKNESS MACULAR HOLES: A COMPARATIVE STUDY IN A LARGE SERIES OF PATIENTS. Retina. 2018;38(Suppl 1):S73-8. https://doi. org/10.1097//AE.0000000000001985.

8. Hayashi H, Kuriyama S. FOVEAL MICROSTRUCTURE IN MACULAR HOLES SURGICALLY CLOSED BY INVERTED INTERNAL LIMITING MEMBRANE FLAP TECHNIQUE. Retina. 2014;34(12):2444-50. https://doi.org/10.1097/IAE. 0000000000000252.

9. Shiode $Y$, Morizane $Y$, Matoba $R$, et al. The role of inverted internal limiting membrane flap in macular hole closure. Invest Opthalmol Vis Sci. 2017; 58(11):4847. https://doi.org/10.1167/iovs.17-21756.

10. Franze $\mathrm{K}$, Grosche J, Skatchkov SN, et al. Müller cells are living optical fibers in the vertebrate retina. Proc Natl Acad Sci U S A. 2007;104(20):8287-92. https://doi.org/10.1073/pnas.0611180104.

11. Kuriyama S, Hayashi $H$, Jingami $Y$, Kuramoto N, Akita J, Matsumoto M. Efficacy of Inverted Internal Limiting Membrane Flap Technique for the Treatment of Macular Hole in High Myopia. Am J Ophthalmol. 2013;156(1): 125-31.e1. https://doi.org/10.1016/j.ajo.2013.02.014.

12. Mete $M$, Alfano A, Guerriero $M$, et al. INVERTED INTERNAL LIMITING MEMBRANE FLAP TECHNIQUE VERSUS COMPLETE INTERNAL LIMITING MEMBRANE REMOVAL IN MYOPIC MACULAR HOLE SURGERY: a comparative study. Retina. 2017:37(10): 1923-30. https://doi.org/10.1097//AE.0000000000001446.

13. Ota $\mathrm{H}$, Kunikata $\mathrm{H}$, Aizawa N, Nakazawa T. Surgical results of internal limiting membrane flap inversion and internal limiting membrane peeling for macular hole. PLoS One. 2018;13(9):e0203789. https://doi.org/10.1371/ journal.pone.0203789.

14. Wakabayashi T, Fujiwara M, Sakaguchi H, Kusaka S, Oshima Y. Fovea microstructure and visual acuity in surgically closed macular holes: spectraldomain optical coherence tomographic analysis. Ophthalmol. 2010;117(9): 1815-24. https://doi.org/10.1016/j.ophtha.2010.01.017.

15. Casini G, Mura M, Figus $M$, et al. INVERTED INTERNAL LIMITING MEMBRANE FLAP TECHNIQUE FOR MACULAR HOLE SURGERY WITHOUT EXTRA MANIPULATION OF THE FLAP. Retina. 2017;37(11):2138-44. https://doi.org/ 10.1097/IAE.0000000000001470.

16. Rossi T, Gelso A, Costagliola C, et al. Macular hole closure patterns associated with different internal limiting membrane flap techniques. Graefes Arch Clin Exp Ophthalmol. 2017;255(6):1073-8. https://doi.org/10. 1007/s00417-017-3598-9.

17. Michalewska Z, Michalewski J, Dulczewska-Cichecka K, Adelman RA, Nawrocki J. TEMPORAL INVERTED INTERNAL LIMITING MEMBRANE FLAP TECHNIQUE VERSUS CLASSIC INVERTED INTERNAL LIMITING MEMBRANE FLAP TECHNIQUE: A comparative study. Retina. 2015;35(9):1844-50. https://doi.org/10.1097//AE.0000000000000555.

18. Ito $\mathrm{Y}$, Terasaki H, Takahashi A, Yamakoshi T, Kondo M, Nakamura M. Dissociated optic nerve fiber layer appearance after internal limiting membrane peeling for idiopathic macular holes. Ophthalmol. 2005;112(8): 1415-20. https://doi.org/10.1016/j.ophtha.2005.02.023.

\section{Publisher's Note}

Springer Nature remains neutral with regard to jurisdictional claims in published maps and institutional affiliations. 